We found a statistically significant lower HIV infection rate among participants aged 20-24 in 2011 compared to 2007 (5.0\% vs $13.4 \%, \mathrm{p}<0.001)$ and among those who reported having ever tested for HIV (3.0\% vs $10.5 \%$, $p=0.02)$.

Conclusion The number and magnitude of differences in the characteristics of the two samples suggests that the two rounds of RDS likely sampled different subsets of the Zanzibar MSM population, limiting their comparability and ability to assess trends over time. Similar findings have been reported with repeated RDS surveys in other settings. Our results highlight the continuing challenge in obtaining representative data among key populations affected by HIV to make evidence-based policy and programme planning decisions. While repeated bio-behavioural cross-sectional surveys using the same methodology in the same population are the backbone of surveillance in key populations, we advocate caution in implementation and interpretation of repeated RDS surveys and that other sampling approaches (e.g., Time Location Sampling) be tested.

\section{P3.326 ESTIMATING THE SIZE OF THE FEMALE SEX WORKER POPULATION IN ASUNCION, PARAGUAY BY MAPPING AND MULTIPLIER ESTIMATES}

doi:10.1136/sextrans-2013-051184.0779

IJ 0 Jacobson, ${ }^{2} \mathbf{G}$ Aguilar, ${ }^{2} \mathrm{~L}$ Gimenez, ${ }^{2} \mathrm{~A}$ Barboza, ${ }^{2} \mathrm{~A}$ Kawabata, ${ }^{2} \mathrm{R}$ Valdez. IIndependent Consultant, Bogota, Colombia, ${ }^{2}$ National Program of Control of HIVAidsSTI, Asuncion, Paraguay

Introduction Population size estimates for most at risk populations are essential for modelling and projections of the HIV epidemic and planning and prioritising prevention interventions. WHO/UNAIDS recommends countries produce multiple estimates given the large variance and biases inherent in available methods.

Methods Between January and May, 2012, 431 female sex workers (FSW) ages 18 and older recruited by time-location sampling participated in a HIV bio-behavioural surveillance survey (BSS) in the greater metropolitan area of Asuncion. Prior to recruitment, sex work venues and public places were mapped and FSW enumerated through key actor interviews. Makeup kits were then distributed to FSW present during site visits to a random sample of mapped venues. BSS question items assessed the percent of study participants that received the kit (the "unique object" method). Additional BSS question items assessed the percent of FSW that had accessed public HIV testing services from July to December, 2011 and services data were obtained on the number of FSW tested at the same sites in the same period (the services multiplier method). Estimated percentages from the BSS were weighted for differential probability of venue selection and incorporated Huber-White adjustments for clustering by venue. Results Mapping identified 425 FSW at 72 sex work locations. Unique objects were distributed to 293 FSW. Of BSS participants, $22.7 \%$ (95\% confidence interval [CI], 17.8\%-28.4\%) reported receiving the object, corresponding to a size estimate of 1292 (IC, 10311644) FSW. 53 FSW accessed HIV testing sites during the period and $6.0 \%$ (IC, $3.8 \%-9.4 \%$ ) of BSS participants reported testing, for a size estimate of 880 (IC, 564-1392) FSW.

Conclusion Size estimates obtained by unique object and services multipliers were not significantly different, while mapping produced a significantly lower estimate, most likely reflecting changes in the population of FSW over time.

\section{P3.327 SURVEILLANCE AND CONTROL OF SEXUALLY TRANSMITTED INFECTIONS IN LITHUANIA}

doi:10.1136/sextrans-2013-051184.0780

${ }^{1} \mathrm{O}$ Ivanauskiene, ${ }^{\mathrm{G}} \mathrm{K}$ Kligys, ${ }^{2} \mathrm{R}$ Butylkina, ${ }^{3} \mathrm{~S}$ Sylvan, ${ }^{4} \mathrm{M}$ Unemo, ${ }^{3} \mathbf{M}$ Domeika, Eastern European Network for SexualReproductive Health. 'Department for Communicable
Diseases Control and Prevention of Kaunas Public Health Centre, Kaunas, Lithuania; ${ }^{2}$ National Public Health Surveillance Laboratory, Vilnius, Lithuania; ${ }^{3}$ Department of Control and Prevention of Communicable Diseases, Uppsala County Council, Uppsala, Sweden; ${ }^{4}$ WHO Collaborating Centre for Gonorrhoea and other STIS, Örebro, Sweden

Background The contemporary national epidemiological surveillance system of sexually transmitted infections in Lithuania was legalised in 2009. A national computerised reporting system has been introduced, aiming to standardise the data collection and analysis for all communicable diseases and their etiological agents through the country.

Methods Analysis of the national database available at www.ulac.lt Results There are four reportable STIs in Lithuania, namely syphilis, Chlamydia trachomatis, Neisseria gonorrhoeae and HIV. Since 2010 both the physicians and the laboratories report identified STI cases. The physician reports individual new STI cases weekly, while the laboratory reports are provided monthly and only as aggregated data. During the period of 2009-2011, the incidences of bacterial STIs in Lithuania have been decreasing. The incidence (cases per 100,000 population) of syphilism decreased from 9.6 to 8.5 cases, of gonorrhoea from 11.5 to 7.9 cases, and of genital C. trachomatis infection from 11.9 to 10.6. In contrast, the incidence of HIV infection during the same time period increased from 0.49 to 5.15 cases! These changes in STI incidences were not due to the level of testing, which has been relatively stable from 2009 to 2011.

Conclusion The substantial increase in HIV incidence during recent years in Lithuania is of major concern. The difference in reporting methodologies, namely reporting of individual cases by the physicians and aggregated data from the laboratory reports does not allow comparison of them sufficiency of such reporting as well as evaluation of the true epidemiological situation.

\section{P3.328 AIDS IN KOSOVO, STIGMA AND KNOWLEDGE AMONG YOUTH}

doi:10.1136/sextrans-2013-051184.0781

'L Gashi, ${ }^{2 D}$ Gexha-Bunjaku, 'N Ramadani. 'National Institute of Public Health of Kosova, Prishtina, Kosovo; ${ }^{2}$ Community Development Fund, Prishtina, Kosovo

Background The official HIV/AIDS data in Kosovo are based on HIV case reporting from health-care services, the blood transfusion system and Voluntary Counselling and Testing centres. Between 1986 and 2012, are reported 87 HIV and AIDS cases, of which 47 were AIDS, $40 \mathrm{HIV}$ and 39 deaths. The majority (69\%) of cases were men, age group 25 to 34 (37\%) and route of transmission is: heterosexual (90\%), MSM (7\%), vertical transmission (2\%) and IDU (1\%). Based on existing data and the UNAIDS classification system, Kosovo is currently still categorised as having a low-level HIV epidemic. Even though with a low HIV prevalence, Kosovo faces a number of threatening factors, including increased number of drug users, a stigmatised and discriminated MSM community, high percentage of youth among general population $(57 \%$ of the population under the age of 25), with changing social norms and especially the sexual ones.

Methods Data collection was done using self administered structured questionnaires amongst 249 high school students. Data were analysed using the Statistical Package for Social Sciences (SPSS).

Results The findings revealed that $68 \%$ of students know that HIV transmission can be reduced by having sex with only one uninfected partner who has no other partners, $94 \%$ know that the risk of getting HIV can be reduced by using a condom every time they have sex, $68 \%$ know that a person cannot get HIV from mosquito bites, $81 \%$ know that they cannot get HIV by sharing food with someone who is infected and $46 \%$ know that a healthy looking person can have HIV. 